\title{
A Stated Preference Experiments for Mobility-as-a-Service Plans
}

\author{
Melinda Matyas and Maria Kamargianni \\ MaaSLab, UCL Energy Institute \\ BSEER, University College London \\ London, UK \\ E-mails: Melinda.matyas.13@ucl.ac.uk \& m.kamargianni@ucl.ac.uk
}

\begin{abstract}
As the Mobility as a Service (MaaS) concept gains wider acceptance, there is increased demand for understanding more about MaaS subscription plans. This paper presents the design of a stated preference (SP) experiment that captures the complex decision making process of purchasing MaaS products. It uses a prompted recall smartphone based travel survey tool that is expanded by a SP experiment regarding MaaS plan choices. Respondents are presented with repeated choices between four hypothetical MaaS plans out of which three are fixed plans and one is a menu option. This approach allows for testing people's preferences and willingness to pay for flexibility. The attributes of the plans include transport modes and amounts, mode specific features (e.g. 10-minute taxi guarantee), transferability (meaning how much of left over mode-attributes can be transferred to the next month), special prizes (e.g. a free dinner for two) and the price of the plan.
\end{abstract}

Keywords: Mobility as a Service; Stated preference design; demand; MaaS plans/packages

\section{INTRODUCTION}

The Mobility as a Service (MaaS) concept has been gaining wider recognition in the mobility sector. Many cities and countries around the world have stated their intentions to introduce this new service model, which restructures the mobility distribution chain to include a MaaS operator who satisfies users' every transportation need through a single digital platform [1]. One of the truly revolutionary elements of MaaS is that it aims to offer travellers the option to buy monthly mobility plans that include a certain amount of each transportation service such that, collectively, they satisfy all their mobility needs [2]. Depending on the local environment, the plans not only include the various public transport options, which in many cities are already offered in monthly subscriptions, but also taxi, car sharing, bike sharing, car rental, long distance rail etc. These plans would conceptually be similar to mobile phone plans, where users pay for a specific amount of services (calls, texts and data) each month.

However, there is still a vast gap in knowledge about the ideal design of the mobility plans. As travellers' needs are immensely heterogeneous, the plans need to be able to cater for the differing preferences of all the socio-demographic user groups. Through the few pilots, projects and thought pieces $[3,4]$ several potential designs have been presented, but there is no quantifiable evidence or consensus about which one would be the best approach. Further, with careful design,
MaaS mobility plans can be used as a travel demand management tool to assist in the shift towards more sustainable travel. Finding subscription plan designs that will both cater to the preferences of users and at the same time support the shift away from this private vehicle dominated is not a straightforward task. Before attempting large-scale MaaS applications it is crucial that some key points are established about user preferences and behaviour. This a priori information can then be used to guide the developments and best practices of MaaS plans.

Against this background, it becomes clear that in depth analysis is needed about preferences and behaviour under various MaaS subscription plan designs. However, to do this, new, currently unavailable data is necessary. As such, in the presented research, stated preference (SP) experiments are introduced and applied to the investigation of the decision making process of MaaS plan choices. The detailed experiments are based upon repeated discrete choices between mobility plans involving the respondents making tradeoffs between the various characteristics and elements of each plan. Information from these has the potential to provide in depth insights into how MaaS plans should be designed. Based on this, the aim of this paper is to present the process of which these SP experiments were created and validated through focus groups. The case study city is London.

\section{STATED PREFERENCE: A REVIEW}

Stated preference techniques are frequently used to gather information about products and services that are not yet available in the market [5]. With discrete choice SP experiments, respondents choose between hypothetical alternatives defined by a set of attributes, where each attribute can take two or more levels. Each alternative will differ in terms of the combination of the attributes' levels. The advantage of using SP over revealed preference data is that it allows the researchers to manipulate the attributes of the choice options and thereby with great speed and statistical efficiency, explore the effect of changes in attributes which could not be otherwise observed [6]. This characteristic makes it an ideal technique to use when there is much yet to discover about a new product, such as Mobility as a Service plans. However, caution needs to be exercised as the number of hypothetical combinations increases exponentially as the 
amount of attributes and levels increases, leading to potential problems.

MaaS plans are a case of product bundling, which is the practice of marketing two or more products or services in a package for a special price [7]. In SP situations such as these, an alternative is defined as a bundle of features [8]. In order to analyse predetermined bundles, studies prompt respondents to state their preferred choices among the proposed bundles. For example, Madden et al. [9] examined broadband delivered entertainment subscription packages based on an experiment where respondents were asked to choose from a list of package options. In some cases, respondents are first shown individual products and then the same products in a package to determine how packaging can change demand. Examples include Hamilton and Koukova [10] who analyse students' perceptions of the relative importance of bundle elements; Fojcic and Proff [11] and Sheng and Pan [12] who both test how bundling could increase product diffusions of a new product; and Janiszewski and Cunha [13] who focus on price discounts in the evaluation of bundles. However, it is not necessary to test respondents' preferences for individual product elements. A widely referenced study by Yadav [14] examines students' choices of magazine subscription bundles based on pairwise comparisons of bundles.

In classic SP situations, the choice task is designed for each person by creating alternatives through a combination of attribute levels from the same finite list, and the respondent's task is to simply pick their preferred option. However, using solely this approach would not be sufficient for us to address the issue of flexible/customisable MaaS subscriptions. Studies that research customizable/flexible product bundles, which are found in the marketing literature, mainly resort to menu based survey designs to determine consumers' preferences. Menu based designs allow respondents to choose their own preferred attribute levels [15]. In some cases, the experiment is designed to include both pre-determine bundles and the choice to customize the elements within the bundle [8, 16, 17]. The widely referenced study by Ben-Akiva and Gershenfeld [8] for example presents 3 fixed packages and 12 individual features to respondents in a study about custom calling product bundles. They allow respondents to choose among: 1 . one fixed package, 2 . one fixed package and any number of extra features, 3. any number of features, 4 . none.

A growing trend in stated preferences is to try to make the respondents choice situations as realistic as possible [18, 19]. This can be especially useful when attribute levels can take a wide range of values, some which may not be relevant for the respondent. When looking at personal mobility choices, this is very true for the amount different individuals use each transportation mode. As such, context aware SP experiments are becoming more frequently applied. Respondent's specific experiences can be incorporated in three ways: (1) by presenting the status quo as an option without detailing the attributes or attribute levels; (2) by including the status quo with all of the information and (3) by presenting alternatives and their levels that are based on the respondents' real life experiences (Rose and Hess, 2009). This latter type, pivotstyle SP experiments use the existing knowledge base of the respondent when creating experiments and is what we will utilize in our SP design [19]. Most such pivot based experiments use preliminary information collected through an RP survey for their reference alternative. Some applications include: Hensher [25], Masiero and Hensher [26] van Cranenburgh et al. [27]. The popularity of these surveys has just more recently taken off, as automation via computers has significantly increased their feasibility.

\section{MAAS STATED PREFENCE DESIGN}

\section{A. Collection Tool}

Before diving into the SP design the data collection tool is discussed. The SP experiment is built as an extension to a prompted recall travel survey that was adapted for the purposes of our study (FMS, [20]). Having access to a state of the art smartphone based travel survey provided a great opportunity to use this tool to enhance the quality and quantity of data collected and available for analysis. It has to be noted, that even though the presented approach is incorporated into this tool, it can also easily be adapted to traditional computer based survey methods. The survey is as follows. After filling out a pre-survey about basic sociodemographic information and details about their current mobility tool choices, respondents are tracked via a smartphone app for a 7-day period. During the span of the tracking, they are reminded to verify their travel and non-travel activities and are asked to answer additional questions about their experiences (completed either on the web interface or their smartphones). Both these questions and questions in the pre-survey focus heavily on usage and attitudes towards the various characteristics of shared modes and innovative services. As the case study area is Greater London, all the elements of the survey are adapted to fit the local environment. After the 7 days of tracking is complete the stated preference (dubbed post-survey) becomes available. First, the revealed preference (RP) data is aggregated and users are presented with a summary record of their mobility behaviour for that week, broken down by transport mode and including information about travel-cost, time, and distance and number of trips. This mobility record will become an important element to build the SP survey.

\section{B. Attributes and Levels}

Now turning to the SP, in choosing attributes, there are many considerations to take into account. First, the factors included should create a realistic choice for the respondent. Second, the number of attributes presented in each experiment should not be too many so that the respondent is able to comprehend the task and make appropriate tradeoffs [21]. Using the above discussed MaaS developments as a basis, attributes fall under two categories: transport mode specific attributes and nonmode specific attributes. The former includes the actual modes contained in the plans as well as the additional features available for each mode, such as including minivan access in car sharing or 10 minute cab guarantee (see Table 1). The 
latter includes characteristics of the plans such as price and transferability of unused elements to next month. The attributes and levels were determined through a priori reasoning and evaluation of the local environment for the case study city. It was noted early on in the design process that there is a significant flexibility-complexity tradeoff; while it would be desirable to include and test all possible modes, service options and innovative concepts, this would be too complex of a cognitive task for the respondents, especially since the whole concept of MaaS is new and needs to be understood.

The assumption was made that if MaaS were introduced it would include only existing transport modes, thus would be built from the current service provisions of the case study city. A dataset was created which included all non-private modes of the city covering characteristics for each mode and supplier such as their business models, pricing structures and subscription possibilities, booking and payment options if applicable and ICT availability (booking apps etc). This dataset was used to determine the attributes used in the SP.

\section{Transport mode specific attributes and levels}

Public transport: Public transport in London includes bus, tube, overground, Docklands Light Rail (DLR), tram, rail and riverboat. Due to the high number of public transport options in the city, it quickly becomes obvious that presenting all of them individually would overcomplicate the public transport attribute. As the transport modes are already integrated with the Oyster card ticketing and payment system they were aggregated and used as "public transport" in the SP. Three public transport levels were chosen: none, unlimited bus and unlimited public transport. These follow the currently existing bus pass and oyster travelcard (unlimited travel) options available in the city. The unlimited pubic transport level had an additional complication. The London travelcards (unlimited public transport) have a zonal aspect to them. Thus, the level had to be 'unlimited public transport in your zones' where the zones were fed from earlier elements of the survey. This tailoring to respondents was crucial as there are huge differences in the prices depending on which zones are included. If the respondent stated that they had a travelcard in the pre-survey, the travelcard zones were fed through from there. If they didn't, the travel diary and tracking was drawn upon, where the Transport for London zones were built into the system using GIS shapefiles and zones where the respondent frequently travelled through were chosen. Further, two other elements had to be taken into account and fed from the pre-survey. Both eligibility for discounts / free travel and disabilities were questions included in the pre-survey and fed into the public transport - and associated price (to be discussed below) - levels.

Bike sharing: In London there is one main bike sharing scheme the Santander Cycles (former Barclays Bikes). The two levels are none and unlimited access for 30 minutes use at a time. This matches up with the current operation of the service. An additional feature was included that allowed for increased bike sharing rental time to 60 minutes at a time.
Similarly to the public transport mode, if the respondent stated in the pre-survey that they have a disability that prevents them from cycling, this mode was excluded.

Taxi: London has a vast amount of taxi services, including the London black cab, ridehailing services (i.e. Uber) and hundreds of minicab companies. A base dataset was created including a selection of these and containing information about their availability, business models and pricing. After evaluating the extent of the taxi provisions, the decision was made to lump all these together to reduce the complexity of the task. Before determining the levels for the taxi attribute, an analysis was conducted on data from the London Travel Demand Survey (LTDS) to get an idea about the ranges people tend to travel using taxi. It quickly became clear, that there are huge differences in these amounts and it is very easy to have levels that are quite far off of what respondents would like to see (this was also tested with focus groups). In order to create better, tailored levels, which will result in improved information gained through the SP, a pivot style design was used. Information from the mobility record about taxi distance travelled on an average month was used as a baseline from which the attribute levels were pivoted off of. This approach had to be slightly altered as respondents with low or null amounts taxi would have little or no variation (or even negative values) in their levels. Analysing some test simulations, 10 miles per month was chosen as the threshold under which pivoting was not used and fixed attribute levels were adopted. There are also some additional feature attributes for taxi, out of which at most one was presented by alternative. These attributes are presented in Table 1.

Car Sharing (Car Clubs): By car sharing (called car clubs in the UK) we mean short term car rental services, where you can pay by the day, hour or in some cases even minute. When mapping out the offerings, differences in the setups were identified. There are two main types of car sharing services available in London: where company owns fleet and when individuals do in community or peer to peer car sharing. We decided to focus our efforts on the first type as the business models of the former tend to be based on individual agreements between the supplier and the customer, thus would be difficult to include in MaaS plans. Six main car sharing operators were identified all with varying geographical coverage. Similarly to the taxi case, pivoting was used here except for those people who don't use car sharing at all, who had predetermined levels. Driving licence possession and disabilities were excluding factors of this attribute. Car sharing also has some additional features, which can not only provide insights into MaaS plans, but also to some characteristics that would encourage more people to use this mode.

Other modes: Other modes, such as car rental, ride sharing and demand responsive transport were also considered but were excluded in the final design. Car rental was excluded, because we wanted to focus on short term, city trips and in London for urban trips car rental is very similar to car sharing. However, it is not as flexible for seamless door-to-door mobility (which is the aim of MaaS) since car rental points are usually in a specific area and users need to travel there to 
collect and return to the cars. Car sharing is much more flexible, even with round trip car sharing, the pick up points are much more dispersed around the city allowing for more options. With the more novel models, such as free floating car sharing which already exists in parts of London, users have complete flexibility. To include longer term hiring, the SP includes car sharing attribute levels on a daily besides the usual hourly levels. This being said, in other cities / areas where car sharing is not available, car rental needs to be included instead. Ride sharing, was falls under the same category as peer to peer car sharing by which they are based on individual agreements between the customer and the supplier. Further, peer to peer as well as demand responsive services are not as well known and in a SP experiment they would need to be explained in much more detail for respondents to understand what they asked to choose about. It needs to be noted that these modes could be integral part of MaaS schemes and future SP experiments should also aim to include these into them.

It has to be pointed out that there is an important focus on the features of taxi and car sharing in this SP plan designs. The reason for this is that these modes are provided by private companies and can offer several innovative features to advance customer experience. These features can be important elements to customising the MaaS user experience.

\section{Non-mode specific attributes and levels}

The non-mode specific attributes that were included in the SP are transferability, special prizes and the price of the plans. Transferability refers to whether left over travel from one month can transferred over to one month or not. It has two levels "none of your credits can be transferred to the next month" and "all of your credits can be transferred to the next month". The special present incentive attribute was included to see whether they can be used as motivational techniques for people to subscribe to certain plans. The hypothesis is that if someone subscribes to a plan that includes public and shared modes that they may have not used before, there is a change they will try it and start using it. Transferability and the special present was not included in the menu as through the focus groups it became clear that the menus had to be kept very simple or respondents will not comprehend the task and will just randomly click through (see Figure 2 below). Regarding price, only the total price of the plans was presented, not the individual price of each element in the plans (including increased prices if mode-specific features were included). This was done so that the respondents would evaluate their willingness to pay for all the elements included in the plans, rather than try to compare each individual unit price. To determine the actual prices presented to the respondents, each mode-specific attribute had a 'base price' that was established though the dataset of all non-private modes in the city. For example, public transport travelcard (monthly pass) costs were taken from Transport for London's pricing tables. As the price of public transport pass prices depend both the travel zones they are valid for as well as the discount pricing bracket (if applicable), in the experiment, these were asked in the pre-survey and identified through the tracking and were fed through directly to the SP. Additionally other information were also fed from earlier parts of the survey, such as disabilities (which excluded certain modes), discounts (e.g. student discounts for public transport), driving licence (if they do not have licences, car sharing option was not available). As many modes have multiple companies offering services, in these cases average values were taken. For each proposed plan, a plan base price was calculated by summing the individual attribute base prices. In the menu options, the prices levels go higher so flexibility can be priced and we can examine respondents' willingness to pay for flexibility.

\section{C. $\quad$ Scenarios Design Testing}

Detailed focus groups coupled with the in depth pre-test work described above lead to a design that is both relevant for MaaS plan research and relatively well understood by respondents. Various design and presentation options were tested and refined in three waves of focus groups. The groups included individuals from a range of sociodemographic backgrounds. The first and the third focus groups were smaller (5 individuals) while the middle one was with around 20 individuals. These took the format of both email feedback and personal interviews (in some cases the combination of both) about preferred design, presented information and wording.

In order to be introduced to the SP exercise, the respondent is presented with the summary of their travel aggregated to a month (hereafter MR; mobility record) as well as a description of Mobility as a Service. An example of this can be seen in Figure 1. The MR shows the distance, time, number of trips and costs for each transport mode the individual uses. The data used to create this is a combination of pre-survey responses, tracking and verification information as well as data gained from integrating external APIs into the system. The example seen in Figure 1 is for a respondent who only walks, takes the bus, the tube and drives his personal vehicle; which is why the the other transport modes have no values. The MR encourages the respondent to start thinking about their overall travel on a monthly basis as well as the concept of multimodality. Through our focus groups, we discovered that subjects found it very exciting to see their travel broken down as such and were very surprised by their statistics. The MR can be a great incentive for respondents to continue with the survey. The wording of the MaaS description proved to be a challenging task. As the concept is new and unknown to the wider community, it had to be explained in terms that were easily understood and relatable. The difficulty came from doing this in a short and concise manner, while making sure all the key MaaS characteristics were included. All three focus groups provided a vast amount of feedback on a number of wording options, which were all taken into account to arrive at the description presented in Figure 1. The wording throughout the SP was put into context - everything was phrased using London terminology and analogies. This approach greatly increased the overall understanding of MaaS as a concept and how it would be relevant to a user. 


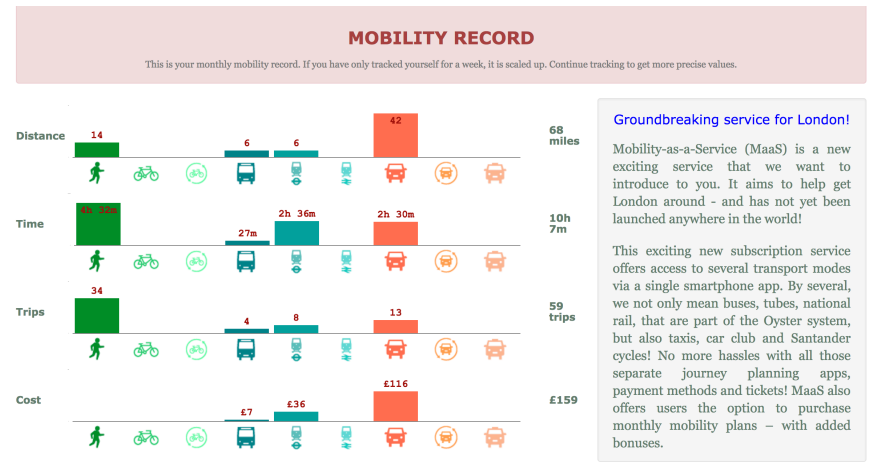

Figure III.1: Introduction to SP

After the introduction page, respondents were presented with a page that included only the following text: 'Now that you have just seen how you tend to travel around London, and you know more about the service, we would like to show you four pages of hypothetical monthly travel plans which we would like to get your opinion on.' During the focus groups, we found that putting this on a separate page rather than under the description of MaaS or straight above the scenarios increased the likelihood of respondents reading and comprehending it.

In each scenario task (page), the respondents were presented with a single choice between 4 different hypothetical plans. The 4 alternatives were: three fixed plans and one menu option where the users can determine which and how much of each mode they would like. These were presented alongside each other, but only one of them can be chosen. Thus, the outcome of a choice made from the options is either one of the three fixed plans or any combination of the individual attributes in the menu option. The menu option is presented alongside the others to allow analysis of the flexibilitycomplexity tradeoffs. Further, the flexibility of the menu option is priced, meaning that the price attribute of the menu is always greater than that of all the other plans. This approach was chosen to allow for analysis of peoples' willingness to pay for flexibility within MaaS plans.

A number of conditions were imposed on each page in order to focus on the research objectives of this study. First, maximum of two 'none' level among the mode-specific attributes was allowed. This had to be imposed as the whole point of MaaS plans is that it offers users a combination of transport modes and if there is only one in the plan the aim is lost. This meant, that for example if a respondent didn't have a driving licence and had a disability where they couldn't cycle, the other two modes (public transport and taxi) had to be included. Another element that we wanted to test was how the additional features affected the choice of plans. Hence, a differentiation was made between two different types of plans: a basic which did not have any additional features and a premium which had additional features. However, even in the premium plans only one additional feature per mode was allowed in order to decrease the cognitive load on respondents. In the first two scenarios there were two basic plans and one premium, in the second two scenarios there were two premiums and one basic. The respondents did not know this distinction. Further, some special features were limited to the premium plans.

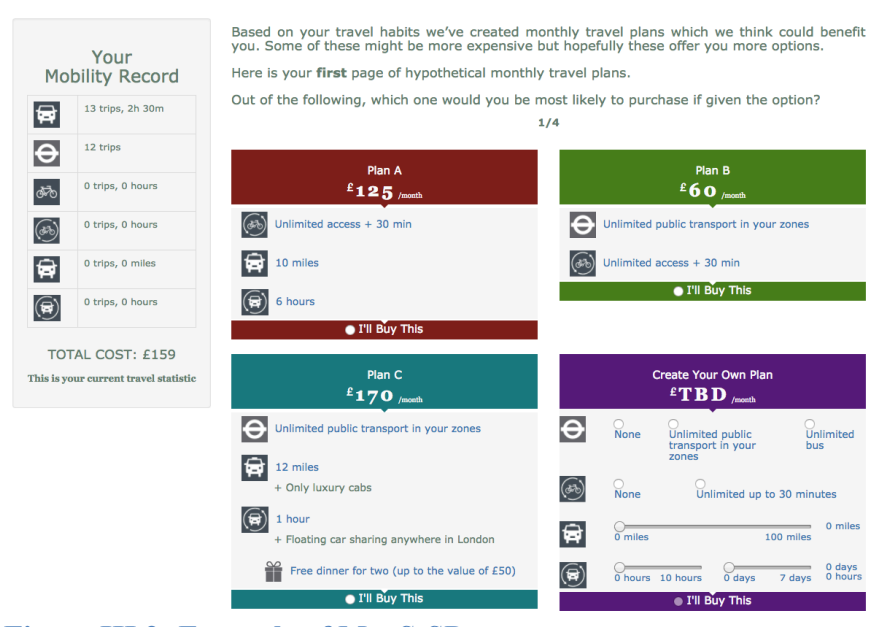

Figure III.2: Example of MaaS-SP

An example of a stated preference experiment presented to the users is depicted in Figure 2. Icons for the travel modes, hover over explanations and colours were used to provide a visually stimulating presentation for respondents. Using pictorial representations makes users' perceptions of modes mode homogenous, makes the task more interesting and easily understandable (Morikawa, 1989). During the focus groups, understanding and acceptance of all the information increased as these elements were included in the design. The icons for the modes were kept the same as those used in the activity diary (tracking/verification) section of the survey that the users had filled out the week before. The order that the modes were presented in remained consistent throughout the mobility records as well as all the plans in each scenario. This way they were familiar with the mode icons and could easily comprehend the main plan concept without having to spend too much time on each page. We preferred this approach as $\mathrm{MaaS}$ is a new concept and we wanted to decrease the effort for the participants.

Each respondent was presented with $4 \mathrm{SP}$ tasks, in which levels were chosen based on a cleaned random experimental design. According to Walker et al. [23] the random design performs as well as any other design and as all designs, will performs even better if it is cleaned to remove choice tasks where one alternative clearly completely dominates the others (hence there is no real tradeoff for the respondents). Hence, there a condition was imposed on the scenarios such that they each had be internally consistent while making sense in with regards to the research topic. If the sum of the base prices of plan $\mathrm{A}$ is greater than the sum or the base prices in plan B, then in the presented alternatives this also has to be true. This method helps minimise the chance of having strictly dominating alternatives, which would be problematic as they may lead to substantially biased estimates [24].

Alongside the plans a short version of the respondents personal MR was presented to give context (see Figure 2). This is similar to a status quo alternative, even though the 
respondent cannot actually choose this. However, after asking the respondents' preference among the 4 plans, they are asked if they would buy their chosen plan if it were available today (the option to use MaaS as pay-as-you-go is also available). Here, they could use their MR as a reference, knowing, that if they would not buy the MaaS plan, they would be picking their status quo.

\section{CONCLUSIONS}

The objective of this study was to present the design of a stated preference experiment that captures the complex decision making process of purchasing MaaS plans. A smartphone based prompted recall travel survey tool was extended with a personalized SP that was built using information about the respondent's actual travel behavior. In the SP, respondents are presented with repeated choices among four hypothetical MaaS plans. Three of the plans are fixed, predetermined bundles, while the fourth is a flexible menu based plan that gives the respondent the opportunity to customize the product. Presenting the two types of plans alongside each other allows research about the differences in preferences and willingness to pay for flexibility. The core attributes and levels of the plans are the amounts of each transport mode, the price of the plan and the transferability, which is the option of carrying over unused travel to next month. Besides these, other features were included as attributes, such as special prizes. The experiments are based upon repeated choices between plans, involving the respondent making tradeoffs between the various characteristics and elements of each plan. The presented design was validated by focus groups and a base MNL was estimated with data from initial waves of data collection.

This research provides the first systematic approach to testing people's preferences towards different Mobility as a Service plan designs using stated preference experiments. The presented design combines several methods, e.g. smartphone activity diaries, pivoting, menu and simple choice into one experiment allowing us to efficiently examine the decisions potential future MaaS users will have to make. Even though the presented survey takes London as a case study, it can be adapted to the local conditions of any other environment. The results of the survey will be able to provide insights into how MaaS plans should be designed and can guide present and future developments.

\section{REFERENCES}

[1] Kamargianni, M., and M. Matyas 2017. The Business Ecosystem of Mobility as a Service. $96^{\text {th }}$ Transportation Research Board (TRB) Annual Meeting, Washington DC, 8-12 January 2017.

[2] Hietanen, Sampo. 'Mobility as a Service' - the New Transport Model? (2016). Eurotransport, Vol 12, Issue 2 ITS \& Transport Management Supplement, pp. 2-4.

[3] Sochor, J., Strömberg, H. and Karlsson, I.M. Implementing mobility as a service: challenges in integrating user, commercial, and societal perspectives. (2015). Transportation Research Record: Journal of the Transportation Research Board, (2536), pp.1-9.

[4] Kamargianni, Maria, Weibo Li, Melinda Matyas, and Andreas Schäfer. A Critical Review of New Mobility Services for Urban Transport. (2016). Transportation Research Procedia Vol. 14, pp. 3294-3303.
[5] Louviere, J.J., Hensher, D.A. and Swait, J.D., 2000. Stated choice methods: analysis and applications. Cambridge University Press.

$\lceil 6\rceil$ Bonnel. P.. Lee-Gosselin. M.. 7mud. I. and Madre. I.L. eds. (2009). Transport survey methods: Keeping up with a changing world. Emerald Group Publishing Limited.

171 Guiltinan. J.P. (1987). The price bundling of services: A normative framework. The Journal of Marketing, pp.74-85.

$\lceil 81$ Ben-Akiva. M. and Gershenfeld. S. (1998). Multi-featured nroducts and services: analvsing nricing and bundling strategies. Journal of Forecasting, 17(3-4), pp.175-196.

[9] Madden, G.G., Simpson, M. and Savage, S. (2002). Broadband delivered entertainment services: forecasting Australian subscription intentions.

[10] Hamilton, R.W. and Koukova, N.T. (2008). Choosing options for nroducts: the effects of mixed bundling on consumers' inferences and choices. Journal of the Academy of Marketing Science, 36(3), pp.423-433.

111 Foicik. T.M. and Proff. H. (2014). Accelerating market diffusion of hatterv electric vehicles through alternative mobilitv concents. International Journal of Automotive Technology and Management 20, 14(3-4), pp.347-368.

$\lceil 121$ Sheng. S. and Pan. Y. (2009). Bundling as a new nroduct introduction strategv: the role of brand image and bundle features. Journal of Retailing and Consumer Services, 16(5), pp.367-376.

「131 Janiszewski. C. and Cunha. M. (2004). The influence of nrice discount framing on the evaluation of a product bundle. Journal of Consumer Research, 30(4), pp.534-546.

$\lceil 141$ Yadav. M.S. (1995). Bundle evaluation in different market segments: The effects of discount framing and buvers' preference heterogeneity. Journal of the Academy of Marketing Science, 23(3), pp.206-215.

[15] Bharati, P., Chaudhury, A. (2004). Using choiceboards to create business value. Communications of the ACM 47 (12), 77-81.

[16] Liechty, J., Ramaswamy, V. and Cohen, S.H. (2001). Choice menus for mass customization: An exnerimental annroach for analvzing customer demand with an apvlication to a web-based information service. Journal of Marketing research, 38(2), pp.183-196.

[17] Moore, C. (2010). Analysing Pick n' Mix Menus via Choice Analysis to Optimise Client Portfolio. Proceedings of the Sawtooth Software Conference, October, 2010

[18] Hensher, D.A. and Greene, W.H. (2003). The mixed logit model: the state of practice. Transportation, 30(2), pp.133-176.

$\lceil 191$ Rose. I. and Hess. S. (2009). Dual-resnonse choices in nivoted stated choice experiments. Transportation Research Record: Journal of the Transportation Research Board, (2135), pp.25-33.

[20] Cottrill, C., Pereira, F., Zhao, F., Dias, I., Lim, H., Ben-Akiva, M. and Zegras, P. (2013). Future mobility survey: Experience in developing a smartphone-based travel survey in Singapore. Transportation Research Record: Journal of the Transportation Research Board, (2354), pp.59-67.

1211 Hensher. D.A. (2006). How do respondents process stated choice exneriments? Attrihute consideration under varying information load. Journal of applied econometrics, 21(6), pp.861-878

[22] Morikawa, T. (1989) Incorporating Stated Preference Data in Travel Demand Analysis, PhD Thesis, Massachusetts Institute of Technology.

[23] Walker, J., Wang, Y., Thorhauge, M. and Ben-Akiva, M. (2015) DEfficient of Deficient? A Robustness Analysis of Experimental Design in a VOT Estimation, in 'Transportation Research Board $94^{\text {th }}$ Annual Meeting'

[24] Bliemer, M.C., Rose, J.M. and Chorus, C. (2014). Dominancy in stated choice surveys and its impact on scale in discrete choice models. 10th International Conference on Transport Survey Methods, Leura, Australia. [25] Hensher DA. (2004). Accounting for stated choice design dimensionality in willingness to pay for travel time savings. J Transp Econ Policy 38(2):425446.

[26] Masiero, L. and Hensher, D.A. (2011). Shift of reference point and implications on behavioral reaction to gains and losses. Transportation, 38(2), pp.249-271.

[27] Van Cranenburgh, S., Chorus, C.G. and Van Wee, B. (2014). Vacation behaviour under high travel cost conditions-a stated preference of revealed preference approach. Tourism Management, 43, pp.105-118. 
